\title{
Characterization of four-qubit states via Bell inequalities
}

\author{
ZHAO Hui $^{1 *}$, ZHANG XingHua ${ }^{1}$, FEI ShaoMing ${ }^{2} \&$ WANG ZhiXi ${ }^{2}$ \\ ${ }^{1}$ College of Applied Sciences, Beijing University of Technology, Beijing 100124, China; \\ ${ }^{2}$ School of Mathematical Sciences, Capital Normal University, Beijing 100048, China
}

Received November 17, 2012; accepted January 14, 2013

A set of Bell inequalities classifying the quantum entanglement of four-qubit states is presented. These inequalities involve only two measurement settings per observer and can characterize fully separable, bi-separable and tri-separable quantum states. In addition, a quadratic inequality of the Bell operators for four-qubit systems is derived.

Bell inequalities, separability, Bell operators

Citation: Zhao H, Zhang X H, Fei S M, et al. Characterization of four-qubit states via Bell inequalities. Chin Sci Bull, 2013, 58: 2334-2339, doi: 10.1007/s11434-0135884-1

The Bell inequality [1] provided the first possibility to distinguish experimentally between quantum-mechanical predictions and those of local realistic models. Derivations of new and stronger Bell inequalities are one of the most important and challenging subjects in quantum information processing. Since Bell's work, there were many important generalizations such as [2-11] and references therein.

The Bell inequalities presented in [12] involve only two measurement settings per observer and can detect perfectly the quantum entanglement of the generalized GHZ states. By using the idea in constructing Bell operators [12], a set of new Bell inequalities are given in [13], which gives rise to a finer classification of the entanglement for three-qubit systems.

The entanglement of four-qubit systems has been treated in terms of Bell inequalities of Mermin-Klyshko type. In [14] the quantum nonlocality of some four-qubit states, the GHZ state, W state, cluster state and the state proposed in [15], has been investigated, towards the optimal violations of the Bell inequality for these states. The classification of entanglement has been also studied in such as [16-19] with linear inequalities for qubit systems and [20] with non-linear inequalities for detecting bi-separable states in arbitrary dimensional quantum systems.

In this work, we study the quantum entanglement of four-

*Corresponding author (email: zhaohui@bjut.edu.cn) qubit systems by using the idea in constructing Bell operators in [12]. We generalize the results of three-qubit systems in [13] to four-qubit systems. It has been shown that the standard Werner-Wolf-Żukowski-Brukner (WWZB) inequalities cannot detect the entanglement of the generalized Greenberger-Horne-Zeilinger (GHZ) states given by $|\psi\rangle=$ $\cos \alpha|0, \ldots, 0\rangle+\sin \alpha|1, \ldots, 1\rangle$ with $0 \leqslant \alpha \leqslant \pi / 4$ [21,22]. However, the Bell operators constructed in the way provided in [12] can detect the entanglement of the generalized GHZ state wholly. Our Bell operators are constructed by using the idea in [12]. The resulted Bell inequalities can distinguish fully separable, bi-separable and tri-separable states of a four-qubit system. Moreover, these linear Bell inequalities involve only two measurement settings per observer. Analytical formulas of the average values of the Bell operators for four-qubit systems are also derived. And a quadratic inequality of the Bell operators for all four-qubit systems has been presented. Explicit geometrical pictures show the relations between the different types of quantum entanglement and the violations of the inequalities.

We fix some notations used in this work. We use the Dirac's symbols throughout this paper. If a quantum system is in one of a number of states $|\psi\rangle_{i}$, where $i$ is an index, with respective probabilities $p_{i}$, then $\left\{p_{i},|\psi\rangle_{i}\right\}$ is called an ensemble of pure states, and the associated density operator for the system is defined by $\rho=\sum_{i} p_{i} \rho_{i}$. The average value of the 
observable $M$ is written $\langle M\rangle=\langle\psi|M| \psi\rangle$.

\section{Classification of four qubits with Bell inequalities}

Consider $N$ parties and allow each of them to choose independently between two dichotomic observables $A_{j}, B_{j}$ for the $j$-th observer, where $A_{j}=\vec{a}_{j} \cdot \vec{\sigma}^{j}$ and $B_{j}=\vec{b}_{j} \cdot \vec{\sigma}^{j}$, with $\vec{\sigma}^{j}=\left(\sigma_{1}{ }^{(j)}, \sigma_{2}{ }^{(j)}, \sigma_{3}{ }^{(j)}\right)$ the Pauli matrices on the $j$-th qubit, and $\vec{a}_{j}=\left(a_{j}^{(1)}, a_{j}^{(2)}, a_{j}^{(3)}\right), \vec{b}_{j}=\left(b_{j}^{(1)}, b_{j}^{(2)}, b_{j}^{(3)}\right)$ the real unit vectors. The quantum mechanical Bell operator on the $N-1$ qubits except for the $i$-th qubit is defined as [12]

$D_{N}^{(i)}=B_{N-1}^{(i)} \otimes \frac{1}{2}\left(A_{i}+B_{i}\right)+I_{N-1} \otimes \frac{1}{2}\left(A_{i}-B_{i}\right), \quad i=1, \ldots, N$,

where $B_{N-1}^{(i)}$ is the Bell operator of WWZB inequalities on the $N-1$ qubits except for the $i$-th qubit,

$$
\begin{aligned}
B_{N-1}^{(i)}= & \frac{1}{2^{N-1}} \sum_{S_{1}, \ldots, S_{N-1}=-1,1} S\left(S_{1}, \ldots, S_{N-1}\right) \\
& \sum_{K_{1}, \ldots, K_{N-1}=1,2} S_{1}^{K_{1}-1} \ldots S_{N-1}^{K_{N-1}-1} \otimes_{j=1}^{N-1} O_{j}\left(K_{j}\right),
\end{aligned}
$$

where $I_{N-1}$ denotes the corresponding identity matrix. For $S\left(S_{1}, \ldots, S_{N-1}\right)=\sqrt{2} \cos \left(\frac{\pi}{4}\left(S_{1}+\cdots+S_{N}-N\right)-\frac{\pi}{4}\right), O_{j}(1)=$ $A_{j}, O_{j}(2)=B_{j}$, one recovers the Mermin-Ardehali-BelinskiiKlyshko (MABK) inequalities [2].

In the following we study the characterization of entanglement for four-qubit systems, $N=4$.

Theorem 1. For fully separable states $\rho$, we have

$$
\left|\left\langle D_{4}{ }^{(i)}\right\rangle\right| \leqslant 1, \quad i=1,2,3,4 .
$$

Proof. A general pure four-qubit state can be written as $|\psi\rangle=\sum_{i, j, k, l=0}^{1} a_{i j k l}|i j k l\rangle$ with normalization $\sum_{i, j, k, l=0}^{1}\left|a_{i j k l}\right|^{2}=$ 1. A mixed four-qubit state can be expressed as $\rho=\sum_{\alpha} p_{\alpha} \rho_{\alpha}$, where $0<p_{\alpha} \leqslant 1, \sum_{\alpha} p_{\alpha}=1, \rho_{\alpha}=\left|\psi_{\alpha}\right\rangle\left\langle\psi_{\alpha}\right|$ are pure states. Due to the linear property of the average values,

$$
\begin{aligned}
\left|\left\langle D_{4}{ }^{(i)}\right\rangle\right| & =\left|\operatorname{tr}\left(\sum p_{\alpha} \rho_{\alpha} D_{4}^{(i)}\right)\right|=\left|\sum p_{\alpha} \operatorname{tr}\left(\rho_{\alpha} D_{4}^{(i)}\right)\right| \\
& \leqslant \sum\left|p_{\alpha} \operatorname{tr}\left(\rho_{\alpha} D_{4}^{(i)}\right)\right| \leqslant \sum\left|\operatorname{tr}\left(\rho_{\alpha} D_{4}^{(i)}\right)\right|,
\end{aligned}
$$

it is sufficient to consider the pure states. The fully separable pure states can be transformed into the form $|\psi\rangle=|0000\rangle$ in suitable bases. Therefore it is direct to verify that

$$
\begin{aligned}
\left|\left\langle D_{4}{ }^{(1)}\right\rangle\right|= & \mid \frac{1}{4}\left(( a _ { 1 } ^ { ( 3 ) } + b _ { 1 } ^ { ( 3 ) } ) \left(-a_{2}^{(3)} a_{3}^{(3)} a_{4}^{(3)}+a_{2}^{(3)} b_{3}^{(3)} b_{4}^{(3)}\right.\right. \\
& \left.+b_{2}^{(3)} a_{3}^{(3)} b_{4}^{(3)}+b_{2}^{(3)} b_{3}^{(3)} a_{4}^{(3)}\right)+\frac{1}{2}\left(a_{1}^{(3)}-b_{1}^{(3)}\right) \mid \leqslant 1 .
\end{aligned}
$$

Similarly one can prove that $\left|\left\langle D_{4}{ }^{(i)}\right\rangle\right| \leqslant 1$ for $i=2,3,4$.

Next we consider the cases of tri-separable states. We denote $\rho_{i j-k-l}$ a tri-separable state of the form $\rho_{i j} \otimes \rho_{k} \otimes \rho_{l}$, in which qubits $i$ and $j$ are entangled, while qubits $k$ and $l$ are separable, $i \neq j \neq k \neq l=1,2,3,4$.

Theorem 2. For any tri-separable states $\rho_{i j-k-l}, i \neq j \neq k \neq$ $l=1,2,3,4$, we have

$$
\left|\left\langle D_{4}{ }^{(i)}\right\rangle\right|=\left|\left\langle D_{4}{ }^{(j)}\right\rangle\right| \leqslant 1, \quad\left|\left\langle D_{4}{ }^{(k)}\right\rangle\right|=\left|\left\langle D_{4}{ }^{(l)}\right\rangle\right| \leqslant \frac{3}{2} .
$$

Proof. We consider the case of $\rho_{12-3-4}$. Every pure state in $\rho_{12-3-4}$ can be written in a Schmidt form, $|\psi\rangle=(\cos \alpha|01\rangle-$ $\sin \alpha|10\rangle) \otimes|0\rangle \otimes|0\rangle$. Therefore

$$
\begin{aligned}
\left|\left\langle D_{4}{ }^{(1)}\right\rangle_{|\psi\rangle}\right|= & \mid \frac{1}{2}\left[-\left(a_{1}^{(3)} a_{2}^{(3)}+a_{2}^{(3)} b_{1}^{(3)}\right)\right. \\
& \left.-\left(\sum_{k=1}^{2} a_{1}^{(k)} a_{2}^{(k)}+a_{2}^{(k)} b_{1}^{(k)}\right) \sin 2 \alpha\right] \\
& \times \frac{1}{2}\left(-a_{3}^{(3)} a_{4}^{(3)}+b_{3}^{(3)} b_{4}^{(3)}\right) \\
& +\frac{1}{2}\left[-\left(a_{1}^{(3)} b_{2}^{(3)}+b_{1}^{(3)} b_{2}^{(3)}\right)\right. \\
& \left.-\left(\sum_{k=1}^{2} a_{1}^{(k)} b_{2}^{(k)}+b_{1}^{(k)} b_{2}^{(k)}\right) \sin 2 \alpha\right] \\
& \times \frac{1}{2}\left(a_{3}^{(3)} b_{4}^{(3)}+b_{3}^{(3)} a_{4}^{(3)}\right) \\
& +\frac{1}{2}\left(a_{1}^{(3)}-b_{1}^{(3)}\right) \cos 2 \alpha \mid \leqslant 1 .
\end{aligned}
$$

Similarly one can prove that $\left|\left\langle D_{4}{ }^{(2)}\right\rangle_{|\psi\rangle}\right| \leqslant 1$. For the Bell operator $D_{4}^{(3)}$, we have

$$
\begin{aligned}
\left|\left\langle D_{4}{ }^{(3)}\right\rangle_{|\psi\rangle}\right|= & \mid \frac{1}{4}\left\{\left[-\left(-a_{1}^{(3)} a_{2}^{(3)}+b_{1}^{(3)} b_{2}^{(3)}\right)\right.\right. \\
& \left.-\left(\sum_{k=1}^{2}-a_{1}^{(k)} a_{2}^{(k)}+b_{1}^{(k)} b_{2}^{(k)}\right) \sin 2 \alpha\right]\left[\left(a_{3}^{(3)}+b_{3}^{(3)}\right) a_{4}^{(3)}\right] \\
& +\left[-\left(a_{1}^{(3)} b_{2}^{(3)}+b_{1}^{(3)} a_{2}^{(3)}\right)\right. \\
& \left.\left.-\left(\sum_{k=1}^{2} a_{1}^{(k)} b_{2}^{(k)}+b_{1}^{(k)} a_{2}^{(k)}\right) \sin 2 \alpha\right]\left[\left(a_{3}^{(3)}+b_{3}^{(3)}\right) b_{4}^{(3)}\right]\right\} \\
& +\frac{1}{2}\left(a_{3}^{(3)}-b_{3}^{(3)}\right) \mid \leqslant \frac{3}{2} .
\end{aligned}
$$

In a similar way, we have $\left|\left\langle D_{4}{ }^{(4)}\right\rangle_{|\psi\rangle}\right| \leqslant \frac{3}{2}$. The cases of $\rho_{1-2-34}, \rho_{13-2-4}, \rho_{1-3-24}, \rho_{14-2-3}$ and $\rho_{1-4-23}$ can be similarly proved. According to the linear property of average values, for all tri-separable states we have (2).

Finally we consider the cases of bi-separable states. There are two classes of bi-separable ones. (i) Two entangled qubits $i$ and $j$ are separable from other entangled qubits $k$ and $l$. For example, we denote $\rho_{12-34}$ the bi-separable state of the form $\rho_{12} \otimes \rho_{34}$, where the qubits 12 and 34 are entangled respectively. (ii) A qubit $i$ is separable from the rest genuine tripartite entangled qubits $j, k$ and $l$. For instance, $\rho_{1-234}$ denotes a bi-separable state of the form $\rho_{1} \otimes \rho_{234}$, where qubits 234 are genuine entangled. 
Theorem 3. For all bi-separable states $\rho$, we have

$$
\left|\left\langle D_{4}{ }^{(i)}\right\rangle_{\rho}\right| \leqslant \frac{3}{2}, \quad i=1,2,3,4
$$

for $\rho$ in class i), and

$$
\left|\left\langle D_{4}{ }^{(i)}\right\rangle_{\rho}\right| \leqslant \sqrt{3}, \quad i=1,2,3,4,
$$

for $\rho$ in class ii).

Proof. We first consider bi-separable states in class i). A pure state in $\rho_{12-34}$ has the following general form, $|\psi\rangle=$ $(\cos \alpha|01\rangle-\sin \alpha|10\rangle) \otimes(\cos \beta|01\rangle-\sin \beta|10\rangle)$. Hence

$$
\begin{aligned}
\left|\left\langle D_{4}{ }^{(1)}\right\rangle_{|\psi\rangle}\right|= & \mid \frac{1}{4}\left\{\left[-\left(a_{1}{ }^{(3)} a_{2}{ }^{(3)}+a_{2}{ }^{(3)} b_{1}{ }^{(3)}\right)\right.\right. \\
& \left.-\left(\sum_{k=1}^{2} a_{1}{ }^{(k)} a_{2}{ }^{(k)}+a_{2}{ }^{(k)} b_{1}{ }^{(k)}\right) \sin 2 \alpha\right] \\
& \times\left[-\left(-a_{3}{ }^{(3)} a_{4}{ }^{(3)}+b_{3}{ }^{(3)} b_{4}{ }^{(3)}\right)\right. \\
& \left.-\left(\sum_{k=1}^{2}-a_{3}{ }^{(k)} a_{4}{ }^{(k)}+b_{3}{ }^{(k)} b_{4}{ }^{(k)}\right) \sin 2 \beta\right] \\
& +\left[-\left(a_{1}{ }^{(3)} b_{2}{ }^{(3)}+b_{1}{ }^{(3)} b_{2}{ }^{(3)}\right)\right. \\
& \left.-\left(\sum_{k=1}^{2} a_{1}{ }^{(k)} b_{2}{ }^{(k)}+b_{1}{ }^{(k)} b_{2}{ }^{(k)}\right) \sin 2 \alpha\right] \\
& \times\left[-\left(a_{3}{ }^{(3)} b_{4}{ }^{(3)}+a_{4}{ }^{(3)} b_{3}{ }^{(3)}\right)\right. \\
& \left.\left.-\left(\sum_{k=1}^{2} a_{3}{ }^{(k)} b_{4}{ }^{\left({ }^{(k)}\right.}+a_{4}{ }^{(k)} b_{3}{ }^{(k)}\right) \sin 2 \beta\right]\right\} \\
& +\frac{1}{2}\left(a_{1}{ }^{(3)}-b_{1}{ }^{(3)}\right) \cos 2 \alpha \mid \leqslant \frac{3}{2} .
\end{aligned}
$$

Similarly one can get $\left|\left\langle D_{4}{ }^{(i)}\right\rangle_{|\psi\rangle}\right| \leqslant \frac{3}{2}$ for $i=2,3,4$. For the cases of $\rho_{13-24}$ and $\rho_{14-23}$, we can also similarly have $\left|\left\langle D_{4}{ }^{(i)}\right\rangle_{|\psi\rangle}\right| \leqslant \frac{3}{2}$ for $i=1,2,3,4$.

For bi-separable states in class ii), we consider the case of $\rho_{1-234}$. There are two inequivalent kinds of genuine threequbit entangled states, the GHZ-type and W-type [23]. For simplicity in the following we denote $c_{x}=\cos x$ and $s_{x}=$ $\sin x$. The GHZ-type state can be written as

$$
\left|\psi_{\mathrm{GHZ}}\right\rangle=\sqrt{K}\left(c_{\delta}|0\rangle|0\rangle|0\rangle+s_{\delta} e^{i \varphi}\left|\phi_{A}\right\rangle\left|\phi_{B}\right\rangle\left|\phi_{C}\right\rangle\right),
$$

where $\left|\phi_{A}\right\rangle=c_{\alpha}|0\rangle+s_{\alpha}|1\rangle, \quad\left|\phi_{B}\right\rangle=c_{\beta}|0\rangle+s_{\beta}|1\rangle, \quad\left|\phi_{C}\right\rangle=$ $c_{\gamma}|0\rangle+s_{\gamma}|1\rangle, \delta \in\left(0, \frac{\pi}{4}\right], \alpha, \beta, \gamma \in\left(0, \frac{\pi}{2}\right], \varphi \in[0,2 \pi)$ and $K=\left(1+2 c_{\delta} s_{\delta} c_{\alpha} c_{\beta} c_{\gamma} c_{\varphi}\right)^{-1} \in\left(\frac{1}{2}, \infty\right)$ is a normalization factor. The W-type state can be written as

$$
\left|\psi_{\mathrm{W}}\right\rangle=\sqrt{a}|001\rangle+\sqrt{b}|010\rangle+\sqrt{c}|100\rangle+\sqrt{d}|000\rangle,
$$

where $a, b, c>0$ and $d=1-(a+b+c) \geqslant 0$. Therefore every pure state in $\rho_{1-234}$ via a suitable choice of bases can be written as $\left|\psi_{0-\mathrm{GHZ}}\right\rangle=|0\rangle \otimes\left|\psi_{\mathrm{GHZ}}\right\rangle$, or $\left|\psi_{0-\mathrm{W}}\right\rangle=|0\rangle \otimes\left|\psi_{\mathrm{W}}\right\rangle$.
We calculate here the value $\left|\left\langle A_{2} A_{3} A_{4}\right\rangle_{\left|\psi_{\mathrm{GHZ}}\right\rangle}\right|$. The other items have similar expressions.

$\left|\left\langle A_{2} A_{3} A_{4}\right\rangle_{\left|\psi_{\mathrm{GHZ}}\right\rangle}\right|=$

$K \mid a_{2}^{(3)} a_{3}^{(3)} a_{4}^{(3)}\left(c_{\delta}^{2}+s_{\delta}^{2} \cdot \cos 2 \alpha \cdot \cos 2 \beta \cdot \cos 2 \gamma\right)$

$+a_{2}^{(1)} a_{3}^{(3)} a_{4}^{(3)}\left(\sin 2 \delta \cdot c_{\varphi} s_{\alpha} c_{\beta} c_{\gamma}+s_{\delta}^{2} \cdot \sin 2 \alpha \cdot \cos 2 \beta \cdot \cos 2 \gamma\right)$

$+a_{2}^{(3)} a_{3}^{(1)} a_{4}^{(3)}\left(\sin 2 \delta \cdot c_{\varphi} c_{\alpha} s_{\beta} c_{\gamma}+s_{\delta}^{2} \cdot \cos 2 \alpha \cdot \sin 2 \beta \cdot \cos 2 \gamma\right)$

$+a_{2}^{(3)} a_{3}^{(3)} a_{4}^{(1)}\left(\sin 2 \delta \cdot c_{\varphi} c_{\alpha} c_{\beta} s_{\gamma}+s_{\delta}^{2} \cdot \cos 2 \alpha \cdot \cos 2 \beta \cdot \sin 2 \gamma\right)$

$+a_{2}^{(1)} a_{3}^{(1)} a_{4}^{(3)}\left(\sin 2 \delta \cdot c_{\varphi} s_{\alpha} s_{\beta} s_{\gamma}+s_{\delta}^{2} \cdot \sin 2 \alpha \cdot \sin 2 \beta \cdot \cos 2 \gamma\right)$

$-a_{2}^{(2)} a_{3}^{(2)} a_{4}^{(3)} \sin 2 \delta \cdot c_{\varphi} s_{\alpha} s_{\beta} c_{\gamma}$

$+a_{2}^{(1)} a_{3}^{(3)} a_{4}^{(1)}\left(\sin 2 \delta \cdot c_{\varphi} s_{\alpha} c_{\beta} s_{\gamma}+s_{\delta}^{2} \cdot \sin 2 \alpha \cdot \cos 2 \beta \cdot \sin 2 \gamma\right)$

$-a_{2}^{(2)} a_{3}^{(3)} a_{4}^{(2)} \sin 2 \delta \cdot c_{\varphi} s_{\alpha} c_{\beta} s_{\gamma}$

$+a_{2}^{(3)} a_{3}^{(1)} a_{4}^{(1)}\left(\sin 2 \delta \cdot c_{\varphi} c_{\alpha} s_{\beta} s_{\gamma}+s_{\delta}^{2} \cdot \cos 2 \alpha \cdot \sin 2 \beta \cdot \sin 2 \gamma\right)$

$-a_{2}^{(3)} a_{3}^{(2)} a_{4}^{(2)} \sin 2 \delta \cdot c_{\varphi} c_{\alpha} s_{\beta} s_{\gamma}$

$\left.+a_{2}^{(1)} a_{3}^{(1)} a_{4}^{(1)} s_{\delta}^{2} \cdot \sin 2 \alpha \cdot \sin 2 \beta \cdot \sin 2 \gamma\right)$

$+\left(a_{2}^{(1)} a_{3}^{(1)} a_{4}^{(1)}-a_{2}^{(2)} a_{3}^{(2)} a_{4}^{(1)}-a_{2}^{(2)} a_{3}^{(1)} a_{4}^{(2)}-a_{2}^{(1)} a_{3}^{(2)} a_{4}^{(2)}\right) \sin 2 \delta$

$\times c_{\varphi} s_{\alpha} s_{\beta} s_{\gamma} \mid$.

$\left|\left\langle A_{2} A_{3} A_{4}\right\rangle_{\left|\psi_{\mathrm{GHZ}}\right\rangle}\right|$ attains its maximum at $\alpha=\beta=\gamma=\frac{\pi}{2}, \varphi=0$ and $\delta=\frac{\pi}{4}$ according to the value of $K$ and the property of the trigonometric functions. Hence we have

$$
\begin{aligned}
& \left|\left\langle D_{4}^{(1)}\right\rangle_{\left|\psi_{0-\mathrm{GHZ}}\right\rangle}\right| \\
\leqslant & \mid \frac{1}{4}\left(a_{1}^{(3)}+b_{1}^{(3)}\right)\left[a_{2}^{(1)}\left(a_{3}^{(1)} a_{4}^{(1)}-a_{3}^{(2)} a_{4}^{(2)}-b_{3}^{(1)} b_{4}^{(1)}+b_{3}^{(2)} b_{4}^{(2)}\right)\right. \\
& +a_{2}^{(2)}\left(-a_{3}^{(1)} a_{4}^{(2)}-a_{3}^{(2)} a_{4}^{(1)}+b_{3}^{(1)} b_{4}^{(2)}+b_{3}^{(2)} b_{4}^{(1)}\right) \\
& +b_{2}^{(1)}\left(-a_{3}^{(1)} b_{4}^{(1)}+a_{3}^{(2)} b_{4}^{(2)}-b_{3}^{(1)} a_{4}^{(1)}+b_{3}^{(2)} a_{4}^{(2)}\right) \\
& \left.+b_{2}^{(2)}\left(a_{3}^{(1)} b_{4}^{(2)}+a_{3}^{(2)} b_{4}^{(1)}+b_{3}^{(1)} a_{4}^{(2)}+b_{3}^{(2)} a_{4}^{(1)}\right)\right] \\
& +\frac{1}{2}\left(a_{1}^{(3)}-b_{1}^{(3)}\right) \mid \leqslant 1 .
\end{aligned}
$$

Using the similar method above, we have also $\left|\left\langle D_{4}{ }^{(i)}\right\rangle_{\left|\psi_{0-\mathrm{GHz}}\right\rangle}\right\rangle$ $\leqslant 1$ for $i=2,3,4$.

Next we compute $\left|\left\langle A_{2} A_{3} A_{4}\right\rangle_{\left|\psi_{\mathrm{w}}\right\rangle}\right\rangle$.

$$
\begin{aligned}
&\left|\left\langle A_{2} A_{3} A_{4}\right\rangle_{\left|\psi_{\mathrm{w}}\right\rangle}\right| \\
&=\mid \mid(d-a-b-c) a_{2}^{(3)} a_{3}^{(3)} a_{4}^{(3)}+2 \sqrt{c d} a_{2}^{(1)} a_{3}^{(3)} a_{4}^{(3)} \\
&+2 \sqrt{b d} a_{2}^{(3)} a_{3}^{(1)} a_{4}^{(3)}+2 \sqrt{a d} a_{2}^{(3)} a_{3}^{(3)} a_{4}^{(1)}+2 \sqrt{b c}\left(a_{2}^{(1)} a_{3}^{(1)} a_{4}^{(3)}\right. \\
&\left.+a_{2}^{(2)} a_{3}^{(2)} a_{4}^{(3)}\right)+2 \sqrt{a c}\left(a_{2}^{(1)} a_{3}^{(3)} a_{4}^{(1)}+a_{2}^{(2)} a_{3}^{(3)} a_{4}^{(2)}\right) \\
&+2 \sqrt{a b}\left(a_{2}^{(3)} a_{3}^{(1)} a_{4}^{(1)}+a_{2}^{(3)} a_{3}^{(2)} a_{4}^{(2)}\right) \mid \\
& \leqslant \frac{1}{2} \mid a_{2}^{(3)}\left(-a_{3}^{(3)} a_{4}^{(3)}+a_{3}^{(1)} a_{4}^{(1)}+a_{3}^{(2)} a_{4}^{(2)}+a_{3}^{(1)} a_{4}^{(3)}+a_{3}^{(3)} a_{4}^{(1)}\right) \\
&+a_{2}^{(1)}\left(a_{3}^{(3)} a_{4}^{(3)}+a_{3}^{(1)} a_{4}^{(3)}+a_{3}^{(3)} a_{4}^{(1)}\right)+a_{2}^{(2)}\left(a_{3}^{(2)} a_{4}^{(3)}+a_{3}^{(3)} a_{4}^{(2)}\right) \mid \\
& \leqslant \frac{1}{2}\left|a_{2}^{(3)}+a_{2}^{(1)}+a_{2}^{(2)}\right| \leqslant \frac{\sqrt{3}}{2}
\end{aligned}
$$


where we have used the Cauchy-Schwarz inequality and the relation $\sqrt[4]{a b c d} \leqslant \frac{a+b+c+d}{4}$, for which the equality holds if and only if $a=b=c=d$. Therefore we have

$$
\left|\left\langle D_{4}{ }^{(1)}\right\rangle_{\left|\psi_{0-\mathrm{w}}\right\rangle}\right| \leqslant \frac{1}{4} \times 2 \times\left(\frac{\sqrt{3}}{2} \times 4\right)=\sqrt{3} .
$$

Similarly one can obtain $\left|\left\langle D_{4}{ }^{(i)}\right\rangle_{\left|\psi_{0-\mathrm{w}}\right\rangle}\right\rangle \leqslant \sqrt{3}$ for $i=2,3,4$.

The cases for $\rho_{2-134}, \rho_{3-124}$ and $\rho_{4-123}$ can be similarly proved.

\section{The quadratic inequality of Bell operator for four qubits}

We derive now an analytical quadratic inequality of the Bell operator for four qubits. The four-qubit states $\rho$ can be written as [24],

$$
\begin{aligned}
\rho= & \frac{1}{16}\left(I \otimes I \otimes I \otimes I+\sum_{i_{1}=1}^{3} Q_{i_{1}}^{(1)} \sigma_{i_{1}}^{(1)} \otimes I \otimes I \otimes I\right. \\
& +\sum_{i_{2}=1}^{3} Q_{i_{2}}^{(2)} I \otimes \sigma_{i_{2}}^{(2)} \otimes I \otimes I+\sum_{i_{3}=1}^{3} Q_{i_{3}}^{(3)} I \otimes I \otimes \sigma_{i_{3}}^{(3)} \otimes I \\
& +\sum_{i_{4}=1}^{4} Q_{i_{4}}^{(4)} I \otimes I \otimes I \otimes \sigma_{i_{4}}^{(4)}+\cdots \\
& \left.+\sum_{i_{1}, i_{2}, i_{3}, i_{4}=1}^{3} Q_{i_{1} i_{2} i_{3} i_{4}}^{(1234)} \sigma_{i_{1}}^{(1)} \otimes \sigma_{i_{2}}^{(2)} \otimes \sigma_{i_{3}}^{(3)} \otimes \sigma_{i_{4}}^{(4)}\right) .
\end{aligned}
$$

Set

$$
\begin{aligned}
& \vec{\alpha}=\left(Q_{1}^{(1)}, Q_{2}^{(1)}, Q_{3}^{(1)}\right), \vec{\beta}=\left(Q_{1}^{(2)}, Q_{2}^{(2)}, Q_{3}^{(2)}\right) \\
& \vec{\gamma}=\left(Q_{1}^{(3)}, Q_{2}^{(3)}, Q_{3}^{(3)}\right), \vec{\varepsilon}=\left(Q_{1}^{(4)}, Q_{2}^{(4)}, Q_{3}^{(4)}\right) \\
& \vec{S}=\left(Q_{111}^{(124)}, Q_{112}^{(124)}, Q_{113}^{(124)}, \ldots, Q_{331}^{(124)}, Q_{332}^{(124)}, Q_{333}^{(124)}\right) \\
& \vec{T}=\left(Q_{111}^{(123)}, Q_{112}^{(123)}, Q_{113}^{(123)}, \ldots, Q_{331}^{(123)}, Q_{332}^{(123)}, Q_{333}^{(123)}\right) \\
& \vec{U}=\left(Q_{111}^{(234)}, Q_{112}^{(234)}, Q_{113}^{(234)}, \ldots, Q_{331}^{(234)}, Q_{332}^{(234)}, Q_{333}^{(234)}\right) \\
& \vec{V}=\left(Q_{111}^{(134)}, Q_{112}^{(134)}, Q_{113}^{(134)}, \ldots, Q_{331}^{(134)}, Q_{332}^{(134)}, Q_{333}^{(134)}\right) \\
& \vec{Q}=\left(Q_{1111}^{(1234)}, Q_{1112}^{(1234)}, Q_{1113}^{(1234)}, \ldots, \ldots, Q_{3331}^{(1234)}, Q_{3332}^{(1234)}, Q_{3333}^{(1234)}\right)
\end{aligned}
$$

We have the following lemma.

Lemma. For four qubits pure states, we have the following equality,

$$
|\vec{\alpha}|^{2}+|\vec{\beta}|^{2}+|\vec{\gamma}|^{2}+|\vec{\varepsilon}|^{2}+|\vec{S}|^{2}+|\vec{T}|^{2}+|\vec{U}|^{2}+|\vec{V}|^{2}+|\vec{Q}|^{2}=9
$$

Proof. A four-qubit pure state $|\psi\rangle$ can be also written as [25],

$$
|\psi\rangle=l_{0}|0000\rangle+l_{1}|0011\rangle+l_{2}|0101\rangle+l_{3}|0110\rangle+l_{4}|1100\rangle
$$

$$
\begin{aligned}
& +l_{5}|1001\rangle+l_{6}|1010\rangle+l_{7}|1011\rangle+l_{8}|0111\rangle+l_{9}|1101\rangle \\
& +l_{10}|1110\rangle+l_{11}|1111\rangle,
\end{aligned}
$$

where $l_{i}$ with $i=7,8,9,10,11$, are non-negative real numbers and $\left|l_{0}\right| \geqslant\left|l_{i}\right|$ for $i=1,2, \ldots, 11$. Comparing $\rho=|\psi\rangle\langle\psi|$ with (5), we have the relation (6) by straightforward calculation.

Here $|\vec{\alpha}|^{2},|\vec{\beta}|^{2},|\vec{\gamma}|^{2},|\vec{\varepsilon}|^{2},|\vec{S}|^{2},|\vec{T}|^{2},|\vec{U}|^{2},|\vec{V}|^{2},|\vec{Q}|^{2}$ are all invariants under local unitary transformations, and equality (6) holds for all pure states. The minimum of $|\vec{Q}|$ is attained for fully separable states and the maximum of $|\vec{Q}|$ is obtained for maximally entangled states.

Theorem 4. Any four-qubit mixed state $\rho$ satisfies the following inequality,

$$
\omega=\left\langle D_{4}{ }^{(1)}\right\rangle_{\rho}^{2}+\left\langle D_{4}{ }^{(2)}\right\rangle_{\rho}^{2}+\left\langle D_{4}{ }^{(3)}\right\rangle_{\rho}^{2}+\left\langle D_{4}{ }^{(4)}\right\rangle_{\rho}^{2} \leqslant 4 .
$$

Proof. Due to that the quadratic function is a convex function,

$$
\begin{aligned}
\left\langle D_{4}{ }^{(i)}\right\rangle^{2} & =\left[\operatorname{tr}\left(\sum p_{\alpha} \rho_{\alpha} D_{4}^{(i)}\right)\right]^{2}=\left[\sum p_{\alpha} \operatorname{tr}\left(\rho_{\alpha} D_{4}^{(i)}\right)\right]^{2} \\
& \leqslant \sum p_{\alpha}\left[\operatorname{tr}\left(\rho_{\alpha} D_{4}^{(i)}\right)\right]^{2} \leqslant \sum\left[\operatorname{tr}\left(\rho_{\alpha} D_{4}^{(i)}\right)\right]^{2},
\end{aligned}
$$

it is sufficient to consider only pure states. Set

$$
\begin{array}{ll}
C_{i}=\frac{1}{2}\left(A_{i}+B_{i}\right), & D_{i}=\frac{1}{2}\left(B_{i}-A_{i}\right), \\
\vec{s}_{i}=\frac{1}{2}\left(\overrightarrow{b_{i}}+\overrightarrow{a_{i}}\right), & \overrightarrow{t_{i}}=\frac{1}{2}\left(\overrightarrow{b_{i}}-\overrightarrow{a_{i}}\right),
\end{array}
$$

We have $\left|\vec{s}_{i}\right|^{2}+\left|\overrightarrow{t_{i}}\right|^{2}=1, \overrightarrow{s_{i}} \cdot \overrightarrow{t_{i}}=0$, and

$$
\begin{aligned}
& \left\langle D_{4}{ }^{(1)}\right\rangle_{\rho}^{2}=\left\{\left[\overrightarrow{s_{1}} \otimes \overrightarrow{s_{2}} \otimes\left(\overrightarrow{s_{3}} \otimes \overrightarrow{t_{4}}-\overrightarrow{t_{3}} \otimes \overrightarrow{t_{4}}+\overrightarrow{s_{3}} \otimes \overrightarrow{s_{4}}+\overrightarrow{t_{3}} \otimes \overrightarrow{s_{4}}\right)\right.\right. \\
& \left.\left.+\overrightarrow{s_{1}} \otimes \overrightarrow{t_{2}} \otimes \overrightarrow{s_{3}} \otimes \overrightarrow{s_{4}}-\overrightarrow{t_{3}} \otimes \overrightarrow{s_{4}}-\overrightarrow{t_{3}} \otimes \overrightarrow{t_{4}}-\overrightarrow{s_{3}} \otimes \overrightarrow{t_{4}}\right)\right] \\
& \left.\vec{Q}-\overrightarrow{t_{1}} \cdot \vec{\alpha}\right\}^{2} \\
& \left\langle D_{4}{ }^{(2)}\right\rangle_{\rho}^{2}=\left\{\left[\overrightarrow{t_{1}} \otimes \overrightarrow{s_{2}} \otimes\left(\overrightarrow{s_{3}} \otimes \overrightarrow{s_{4}}-\overrightarrow{t_{3}} \otimes \overrightarrow{s_{4}}-\overrightarrow{s_{3}} \otimes \overrightarrow{t_{4}}-\overrightarrow{t_{3}} \otimes \overrightarrow{t_{4}}\right)\right.\right. \\
& +\overrightarrow{s_{1}} \otimes \overrightarrow{s_{2}} \otimes \overrightarrow{s_{3}} \otimes \overrightarrow{t_{4}}-\otimes \overrightarrow{t_{3}} \otimes \overrightarrow{t_{4}}+\overrightarrow{s_{3}} \otimes \overrightarrow{s_{4}} \\
& \left.\left.\left.+\overrightarrow{t_{3}} \otimes \overrightarrow{s_{4}}\right)\right] \cdot \vec{Q}-\overrightarrow{t_{2}} \cdot \vec{\beta}\right\}^{2}
\end{aligned}
$$

where $\vec{s} \otimes \vec{t} \otimes \vec{p} \otimes \vec{q} \cdot \vec{Q}$ denotes $\sum_{i j k h} s_{i} t_{j} p_{k} q_{h} Q_{i j k h} . \omega$ attains its maximum at either $|\vec{Q}|=1$ or $|\vec{Q}|=3$. For the case of $|\vec{Q}|=1,|\psi\rangle$ is fully separable and the inequality is satisfied by using Theorem 1 . For the case of $|\vec{Q}|=3$, $|\psi\rangle$ is maximally entangled. Without losing generality, we consider the maximally entangled state $|\psi\rangle=\frac{1}{\sqrt{2}}(|0000\rangle+|1111\rangle)$. Since 
$Q_{1111}=Q_{2222}=Q_{3333}=1, Q_{1122}=Q_{1212}=Q_{2112}=$ $Q_{2121}=Q_{1221}=Q_{2211}=-1$, the rest $Q_{i j k h}=0$ and $\left|\vec{s}_{i}\right|^{2}$ $+\left|\vec{t}_{i}\right|^{2}=1, \vec{s}_{i} \cdot \vec{t}_{i}=0$, thus to attain the maximum of $\omega$, the third components of $\vec{s}_{i}$ and $\overrightarrow{t_{i}}$ should be zero, and either $\left|\vec{s}_{i}\right|=\left|\overrightarrow{t_{i}}\right|$ or one of the $\left|\vec{s}_{i}\right|$ and $\left|\overrightarrow{t_{i}}\right|$ is zero and the other one is 1. Let $i \neq j \neq k \neq l \in\{1,2,3,4\}$, we can obtain the following classifications:

(1) $\left|\vec{s}_{i}\right|=1\left(0\right.$ or $\left.\frac{1}{\sqrt{2}}\right),\left|\vec{s}_{j}\right|=\left|\vec{s}_{k}\right|=\left|\vec{s}_{l}\right|=1 \quad\left(0\right.$ or $\left.\frac{1}{\sqrt{2}}\right)$,

(2) $\left|\vec{s}_{i}\right|=\left|\vec{s}_{j}\right|=0,\left|\vec{s}_{k}\right|=\left|\vec{s}_{l}\right|=\frac{1}{\sqrt{2}}$,

(3) $\left|\vec{s}_{i}\right|=\left|\vec{s}_{j}\right|=1,\left|\overrightarrow{s_{k}}\right|=\left|\vec{s}_{l}\right|=0\left(\right.$ or $\left.\frac{1}{\sqrt{2}}\right)$,

(4) $\left|\vec{s}_{i}\right|=\left|\vec{s}_{j}\right|=1,\left|\overrightarrow{s_{k}}\right|=0,\left|\overrightarrow{s_{l}}\right|=\frac{1}{\sqrt{2}}$,

(5) $\left|\vec{s}_{i}\right|=\left|\vec{s}_{j}\right|=0,\left|\vec{s}_{k}\right|=1,\left|\vec{s}_{l}\right|=\frac{1}{\sqrt{2}}$,

(6) $\left|\vec{s}_{i}\right|=\left|\vec{s}_{j}\right|=\frac{1}{\sqrt{2}},\left|\vec{s}_{k}\right|=0, \quad\left|\vec{s}_{l}\right|=1$.

For the case $\left|\overrightarrow{s_{1}}\right|=\left|\overrightarrow{s_{2}}\right|=\left|\overrightarrow{s_{3}}\right|=\left|\overrightarrow{s_{4}}\right|=1$, we have

$\omega=4\left(\overrightarrow{s_{1}} \otimes \overrightarrow{s_{2}} \otimes \overrightarrow{s_{3}} \otimes \overrightarrow{s_{4}} \cdot \vec{Q}\right)^{2}=4\left\langle\psi\left|C_{1} C_{2} C_{3} C_{4}\right| \psi\right\rangle^{2} \leqslant 4$.

For the case $\left|\overrightarrow{s_{1}}\right|=\left|\overrightarrow{s_{2}}\right|=\left|\overrightarrow{s_{3}}\right|=1$ and $\left|\overrightarrow{s_{4}}\right|=\frac{1}{\sqrt{2}}$, we get

$$
\begin{aligned}
\omega= & 3\left(\overrightarrow{s_{1}} \otimes \overrightarrow{s_{2}} \otimes \overrightarrow{s_{3}} \otimes\left(\overrightarrow{t_{4}}+\overrightarrow{s_{4}}\right) \cdot \vec{Q}\right)^{2} \\
& +\left(\overrightarrow{s_{1}} \otimes \overrightarrow{s_{2}} \otimes \overrightarrow{s_{3}} \otimes \overrightarrow{s_{4}} \cdot \vec{Q}\right)^{2} \\
= & 3\left\langle\psi\left|C_{1} C_{2} C_{3}\left(D_{4}+C_{4}\right)\right| \psi\right\rangle^{2} \\
+ & \left\langle\psi\left|C_{1} C_{2} C_{3} C_{4}\right| \psi\right\rangle^{2} \leqslant 4 .
\end{aligned}
$$

For the case $\left|\overrightarrow{s_{1}}\right|=1$ and $\left|\overrightarrow{s_{2}}\right|=\left|\overrightarrow{s_{3}}\right|=\left|\overrightarrow{s_{4}}\right|=\frac{1}{\sqrt{2}}$, using the orthogonal relation of $\overrightarrow{s_{i}}$ and $\overrightarrow{t_{i}}$, we can express $\overrightarrow{s_{i}}$ and $\overrightarrow{t_{i}}$ as

$$
\begin{aligned}
\overrightarrow{s_{1}} & =\left(\sin \theta_{1} \cos \varphi_{1}, \sin \theta_{1} \sin \varphi_{1}, \cos \theta_{1}\right), \\
\vec{s}_{i} & =\frac{1}{\sqrt{2}}\left(\sin \theta_{i} \cos \varphi_{i}, \sin \theta_{i} \sin \varphi_{i}, \cos \theta_{i}\right), \\
\overrightarrow{t_{i}} & =\frac{1}{\sqrt{2}}\left(\cos \theta_{i} \cos \varphi_{i}, \cos \theta_{i} \sin \varphi_{i},-\sin \theta_{i}\right),
\end{aligned}
$$

where $i=2,3,4$. After straightforward calculations, we have

$$
\begin{aligned}
\omega= & {\left[\frac{1}{2 \sqrt{2}} \sin \left(\theta_{1}+\varphi_{1}+\theta_{2}+\varphi_{2}+\theta_{3}+\varphi_{3}+\theta_{4}+\varphi_{4}\right)\right.} \\
& \left.-\cos \left(\theta_{1}+\varphi_{1}+\theta_{2}+\varphi_{2}+\theta_{3}+\varphi_{3}+\theta_{4}+\varphi_{4}\right)\right] \\
& +\frac{4}{2 \sqrt{2}} \cos \theta_{1} \cos \theta_{4}\left[\cos \left(\theta_{2}+\theta_{3}\right)-\sin \left(\theta_{2}+\theta_{3}\right)\right]^{2} \\
\leqslant & 4 .
\end{aligned}
$$

For the case $\left|\overrightarrow{s_{1}}\right|=\left|\overrightarrow{s_{2}}\right|=\left|\overrightarrow{s_{3}}\right|=\left|\overrightarrow{s_{4}}\right|=\frac{1}{\sqrt{2}}$, one can set

$$
\begin{aligned}
\overrightarrow{s_{i}} & =\frac{1}{\sqrt{2}}\left(\sin \theta_{i} \cos \varphi_{i}, \sin \theta_{i} \sin \varphi_{i}, \cos \theta_{i}\right), \\
\overrightarrow{t_{i}} & =\frac{1}{\sqrt{2}}\left(\cos \theta_{i} \cos \varphi_{i}, \cos \theta_{i} \sin \varphi_{i},-\sin \theta_{i}\right)
\end{aligned}
$$

for $i=1,2,3,4$, and obtain $\omega \leqslant 4$. For other cases inequality (7) can be similarly proved.

The inequalities in Theorems 1 to 4 give rise to an explicit geometric picture. Taking the average of $D_{4}^{(1)}, D_{4}^{(2)}, D_{4}^{(3)}$ and $D_{4}^{(4)}$ as the four coordinates of a four dimensional space, from Theorem 4 we have that $\left\langle D_{4}{ }^{(i)}\right\rangle, i=1, \ldots, 4$, constitute a 3-dimensional sphere. All fully separable states are confined in the center, in a 4-dimensional rectangular with size $1 \times 1 \times 1 \times 1$, see Figure 1. While from the Theorem 2, the tri-separable state, for example, $\rho_{12-3-4}$, is in a 4-dimensional rectangular with the size $1 \times 1 \times 3 / 2 \times 3 / 2$. From the Theorem 3 , the bi-separable state in class $i$ ), for example $\rho_{12-34}$, is in a 4 -dimensional rectangular with size $3 / 2 \times 3 / 2 \times 3 / 2 \times 3 / 2$. For the bi-separable state in class $i i$ ), for example $\rho_{1-234}$, we have a 4-dimensional rectangular with size $\sqrt{3} \times \sqrt{3} \times \sqrt{3} \times \sqrt{3}$.

\section{Conclusions and discussion}

We have investigated the classification of four-qubit entanglement in terms of Bell inequalities that involving only two measurement settings per observer. And a quadratic inequality of Bell operator for four-qubit systems has been obtained. The Bell inequalities satisfied by fully separable, bi-separable and tri-separable states of four-qubit systems are analytically derived. Our approach and some of the obtained formulas can be directly generalized to multipartite qubit systems.

However, our inequalities are not both sufficient and necessary for separability of general four-qubit states. The separability problem in terms of Bell inequalities has been solved only for two-qubit case, any pure entangled two-qubit states violate the CHSH inequality [26], as well as the three-qubit case where Chen et al. [27] showed that all pure entangled three-qubit states violate a Bell inequality. For mixed fourqubit systems, the separability problem remains open. Endrejat et al. [28] discussed the relations between optimization operators and combination of the global entanglement measures. One may conjecture that to make the inequalities sufficient conditions for separability of a four-qubit mixed state,

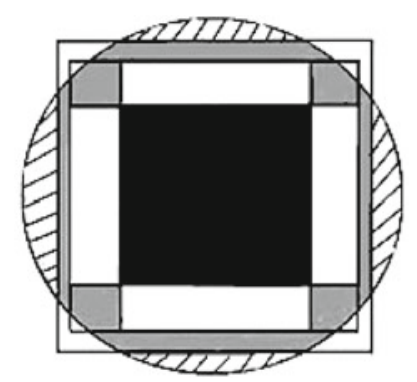

Figure 1 Projection of the state space onto the plane constituted by $\left\langle D_{4}^{(1)}\right\rangle$ and $\left\langle D_{4}^{(3)}\right\rangle$. The fully separable states are in the black region. The triseparable states $\rho_{12-3-4}$ and $\rho_{14-2-3}$ are located inside the white areas respectively between black and gray areas. The bi-separable states $\rho_{12-34}$, $\rho_{14-23}, \rho_{1-234}$ and $\rho_{3-124}$ are inside the gray area. The genuine entangled states are located in the designated slash regions. 
in addition to our Bell operators, some new Bell operators are needed.

H. Zhao acknowledges discussions with Ming Li. This work was supported by the National Natural Science Foundation of China (11101017 and 11275131), Beijing Natural Science Foundation Program and Scientific Research Key Program of Beijing Municipal Commission of Education (KZ201210028032).

1 Bell J S. On the Einstein-Podolsky-Rosen paradox. Physics, 1964, 1: 195-200

2 Gisin N, Bechmann-Pasquinucci H. Bell inequality, Bell states and maximally entangled states for $n$ qubits. Phys Lett A, 1998, 246: 1-6

3 Paterek T, Laskowski W, Żukowski M. On series of multiqubit Bell inequalities. Mod Phys Lett A, 2006, 21: 111-126

4 Yu S X, Chen Q, Zhang C J, et al. All entangled pure states violate a single Bell's inequality. Phys Rev Lett, 2012, 109: 120402-120406

5 Pal K F, Vertesi T. Multisetting Bell-type inequalities for detecting genuine tripartite entanglement. Phys Rev A, 2011, 83: 062123-062129

6 Li M, Fei S M, Li-Jost X Q. Bell inequality, separability and entanglement distillation. Chin Sci Bull, 2011, 56: 945-954

7 Di Y M, Liu S P, Liu D D. Entanglement for a two-parameter class of states in a high-dimension bipartite quantum system. Sci China Phys Mech Astron, 2010, 53: 1868-1872

8 Guo Y, Qi X F, Hou J C. Sufficient and necessary conditions of separability for bipartite pure states in infinite-dimensional systems. Chin Sci Bull, 2011, 56: 840-846

9 Li X K, Li J L, Liu B, et al. The parametric symmetry and numbers of the entangled class of $2 \times M \times N$ system. Sci China Phys Mech Astron, 2011, 54: 1471-1475

10 Wang Y Z, Hou J C, Guo Y. An entanglement criterion for states in infinite-dimensional multipartite quantum systems. Chin Sci Bull, 2012, 57: $1643-1647$

11 He Q Y, Cavalcanti E G, Reid M D, et al. Bell inequalities for Continuous-Variable Measurements. Phys Rev A, 2010, 81: 062106062120

12 Chen K, Albeverio S, Fei S M. Two-setting Bell inequalities for many qubits. Phys Rev A, 2006, 74: 050101-050104(R)
13 Sun B Z, Fei S M. Bell inequalities classifying biseparable three-qubit states. Phys Rev A, 2006, 74: 032335-032338

14 Wu C F, Yeo Y, Kwek L C, et al. Quantum nonlocality of four-qubit entangled states. Phys Rev A, 2007, 75: 032332-032337

15 Yeo Y, Chua W K. Teleportation and dense coding with genuine multipartite entanglement. Phys Rev Lett, 2006, 96: 060502-060505

16 Yu S X, Pan J W, Chen Z B, et al. Comprehensive test of entanglement for two-level systems via the indeterminacy relationship. Phys Rev Lett, 2003, 91: 217903-217906

17 Nagata K, Koashi M, Imoto N. Configuration of separability and tests for multipartite entanglement in Bell-type experiments. Phys Rev Lett, 2002, 89: 260401-260404

18 Brunner N, Sharam J, Vertesi T. Testing the structure of multipartite entanglement with Bell inequalities. Phys Rev Lett, 2012, 108: 110501110505

19 Chen J L, Deng D L, Su H Y, et al. Detecting full $N$-particle entanglement in arbitrarily high-dimensional systems with Bell-type inequality. Phys Rev A, 2011, 83: 022316-022321

20 Nha H, Zubairy M S. Uncertainty inequalities as entanglement criteria for negative partial-transpose states. Phys Rev Lett, 2008, 101: 130402130405

21 Scarani V, Gisin N. Spectral decomposition of Bell's operators for qubits. J Phys A, 2001, 34: 6043-6053

22 Żukowski M, Brukner Č. Laskowski W, et al. Do all pure entangled states violate Bells inequalities for correlation functions? Phys Rev Lett, 2002, 88: 210402-210405

23 Dür W, Vidal G, Cirac J I. Three qubits can be entangled in two inequivalent ways. Phys Rev A, 2000, 62: 062314-062325

24 Linden N, Popescu S, Sudbery A. Nonlocal parameters for multiparticle density matrices. Phys Rev Lett, 1999, 83: 243-247

25 Carteret H A, Higuchi A, Sudbery A. Multipartite generalisation of the Schmidt decomposition. J Math Phys, 2000, 41: 7932-7939

26 Gisin N. Bell's inequality holds for all non-product states. Phys Lett A, 1991, 154: 201-202

27 Chen J L, Wu C F, Kwek L C, et al. Gisin's theorem for three qubits. Phys Rev Lett, 2004, 93: 140407-140410

28 Endrejat J, Büttner H. Characterization of entanglement of more than two qubits with Bell inequalities and global entanglement. Phys Rev A, 2005, 71: 012305-012313

Open Access This article is distributed under the terms of the Creative Commons Attribution License which permits any use, distribution, and reproduction in any medium, provided the original author(s) and source are credited. 